\title{
Implementing a Low-Threshold Analysis with the Askaryan Radio Array (ARA)
}

\author{
Kaeli Hughes ${ }^{a, *}$ on behalf of the ARA Collaboration \\ (a complete list of authors can be found at the end of the proceedings) \\ ${ }^{a}$ The University of Chicago, \\ Chicago, IL, USA \\ E-mail: kahughes@uchicago.edu
}

The Askaryan Radio Array (ARA) is a ground-based radio detector at the South Pole designed to capture Askaryan emission from ultra-high energy neutrinos interacting within the Antarctic ice. The newest ARA station has been equipped with a phased array trigger, in which radio signals in multiple antennas are summed in predetermined directions prior to the trigger. In this way, impulsive signals add coherently, while noise likely does not, allowing the trigger threshold to be lower than a traditional ARA station. Early results on just a fraction of available data from this new system prove the feasibility of a low-threshold analysis.

$37^{\text {th }}$ International Cosmic Ray Conference (ICRC 2021)

July 12 th - 23rd, 2021

Online - Berlin, Germany

\footnotetext{
*Presenter
} 


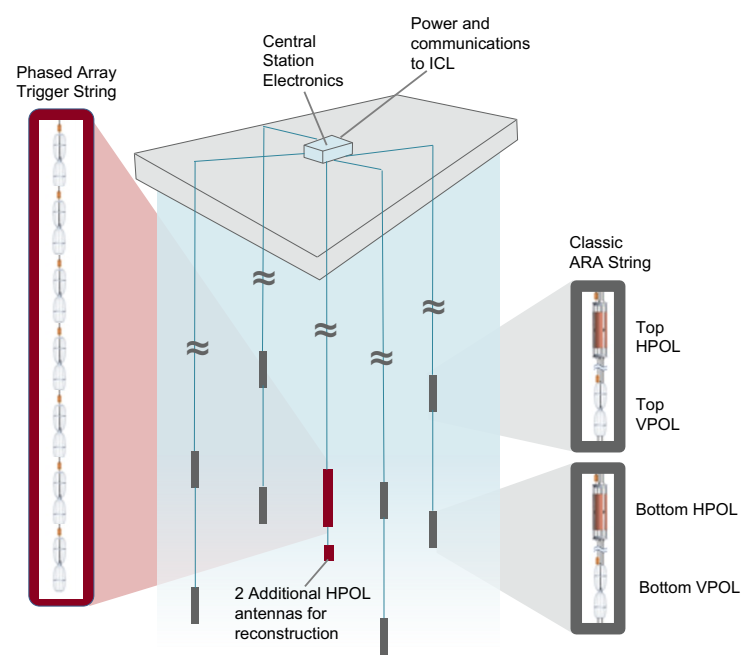

Figure 1: A station diagram of ARA Station 5, including the central phased array trigger string.

\section{Introduction}

Ultra-high energy neutrinos have a unique ability to carry information about their sources from the distant universe. While cosmic rays bend in the presence of magnetic fields, neutrinos travel directly from their sources to detectors, giving neutrino experiments the opportunity to point back at the specific sources in the universe. IceCube has made significant progress in mapping the neutrino flux up to a few PeV [13]; however, the higher energy neutrino flux has not yet been detected.

To be sensitive to the higher energy neutrino flux, the radio detection mechanism has been employed by many experiments, such as RICE [8], ANITA [11], ARIANNA [14], and, covered in this work, ARA [12]. When neutrinos interact in a dense, dielectric medium such as ice, they induce a particle shower. Due to Compton scattering and positron annihilation, the shower takes on a negative overall charge. When this charge travels faster than the speed of light within the medium, Cherenkov emission occurs, which is coherent for wavelengths greater than the lateral width of the shower. In ice, the width of the shower is about $10 \mathrm{~cm}$, leading to coherent emission in radio. This effect, known as the Askaryan Effect, was first theorized in 1961 [1] and was confirmed in 2001 [3].

This work will discuss the status of the most recent ARA analysis and the efficiency improvements made possible by a phased array trigger design.

\section{Instrument Description}

ARA Station 5, depicted in Figure 1, consists of two separate, but connected, experiments, each with their own DAQ box and trigger design. The details of each experiment are in the following sections.

\subsection{The Traditional ARA Trigger}

All five currently deployed traditional ARA stations share the same design: sixteen antennas deployed on four strings, buried up to a depth of 160-200 m. The strings for ARA Station 5 are each separated by $40 \mathrm{~m}$. Events are triggered if three or more antennas register a signal five times 
above the ambient thermal noise level within a time window corresponding to the width of the array. More details on this trigger design can be found in [12]. ARA Station 5 is additionally equipped with a secondary beamforming trigger, referred to as the Phased Array.

The traditional ARA channels can also be read out if the phased array (described below) triggers on an event and the ARA DAQ is not already busy. In this way, there are four possible ways an event could trigger: only the traditional ARA trigger; only the phased array trigger; both systems triggering separately on the same event; or, the phased array triggers and ARA saves the event. About $85 \%$ of phased array triggers are saved by ARA, although many events with identifiable impulsive signals (such as surface events) trigger both systems separately.

\subsection{The Phased Array Trigger}

As discussed in [10], the phased array consists of a single string of seven vertically-polarized antennas, which make up the trigger, and two horizontally-polarized antennas for reconstruction. These antennas are compactly deployed with 1-2 m spacing at a depth of $170 \mathrm{~m}$ in the ice. The Phased Array trigger has fourteen pre-defined beams, each made up of a set of time delays that correspond to different incoming signal directions. By adding the signals together in each beam prior to the trigger, the SNR of real signals improves, as real signals add coherently while noise likely does not. In this way, the phased array is capable of triggering on lower SNR pulses than a traditional ARA station.

\subsection{Livetime}

The livetime included in this study includes the period during 2019 where both the phased array and ARA5 were taking data, equal to approximately 208 days (only $25 \%$ of the total livetime of the phased array so far). This short livetime is due to operating temperature concerns and in-situ testing of a newly deployed surface trigger on the phased array, as well as a USB port failure on the ARA DAQ that occurred in early November. In a typical operating year, the phased array is expected to operate for $>75 \%$ of the year, and is only turned off to avoid overheating in the summer.

\subsection{Simulation}

A modified version of AraSim, discussed in detail in [7], was used to model the phased array trigger. In this simplified trigger, starting with a signal based on Monte-Carlo truth, the signal-tonoise ratio (SNR) of each event was calculated and then assigned a weight based on the expected efficiency as reported on in [10]. The expected efficiency was also weighted based on whether or not the event was directly in a beam. This way of simulating the trigger produces effective volumes that are consistent with real-phasing simulation to within $15 \%$. For comparison against data, the neutrinos were simulated with a flux based on a mixed composition of galactic cosmic rays and high proton energy from the Kotera model [5].

\section{Data Analysis}

A pre-scaling blind analysis method was applied, following the recommendations in [4], in which $10 \%$ of the data was unblinded by choosing one event randomly out of every 10 events. In 
this way, the entire livetime was sampled so that any time-dependent events (such as anthropogenic backgrounds from noisy periods) could be identified easily. This fraction of data was used to set cuts and estimate expected backgrounds, which are defined below.

One advantage of the phased array trigger is that all antennas are constrained to one dimension: a vertical line spanning approximately $10 \mathrm{~m}$. This azimuthal symmetry lends itself to zenith reconstruction. While other ARA stations reconstruct the directions of their events by mapping onto three-dimensional, spherical correlation maps generated at a few specific radii, the phased array instead can produce two-dimensional distance vs. depth maps that plot out the potential path the signal traveled before arriving at the array and includes all available pointing information. An example of such a map is shown in Figure 2.

These correlation maps are used to generate many analysis variables by choosing the bestcorrelating point on the map and adding the signals together to create a coherently summed waveform. Quantities such as the signal-to-noise ratio of the coherently summed waveform, the maximum power, and the impulsivity (calculated using the method from [9] are then saved for every event.

\subsection{Defining A Deep Region}

Outside of thermal noise events, the main sources of background for neutrinos are anthropogenic noise and cosmic ray events, both of which point to the surface, so it is advantageous to separate those coming from deep within the ice. To accomplish this, events can be split into two regions based on the direction of reconstruction and the correlation value when the source hypothesis is placed near the surface. The two variables used to distinguish between deep vs. shallow regions are shown in Figure 3, for both events observed in the 10\% sample and for a simulated flux. The first variable, zenith angle at the antenna, is an indicator of a surface event. This is because the index of refraction changes as a function of depth, leading to only certain zenith angles being physically

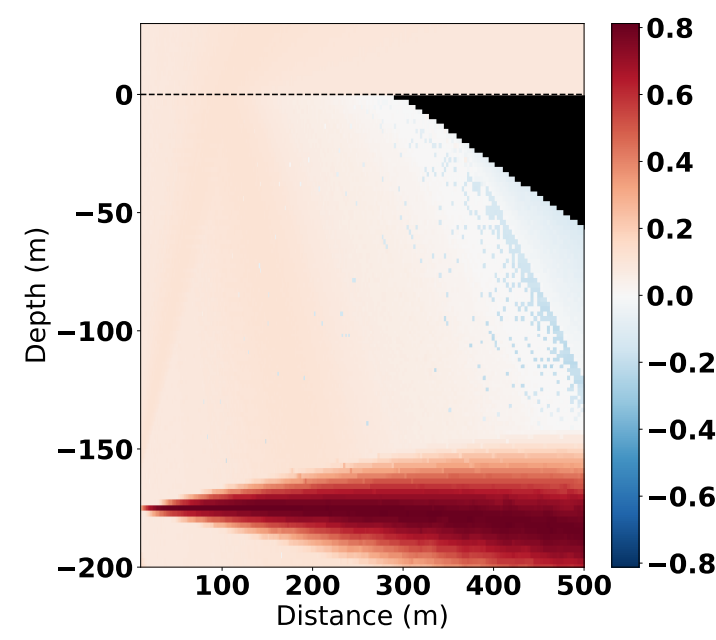

(a) A local cal pulser event.

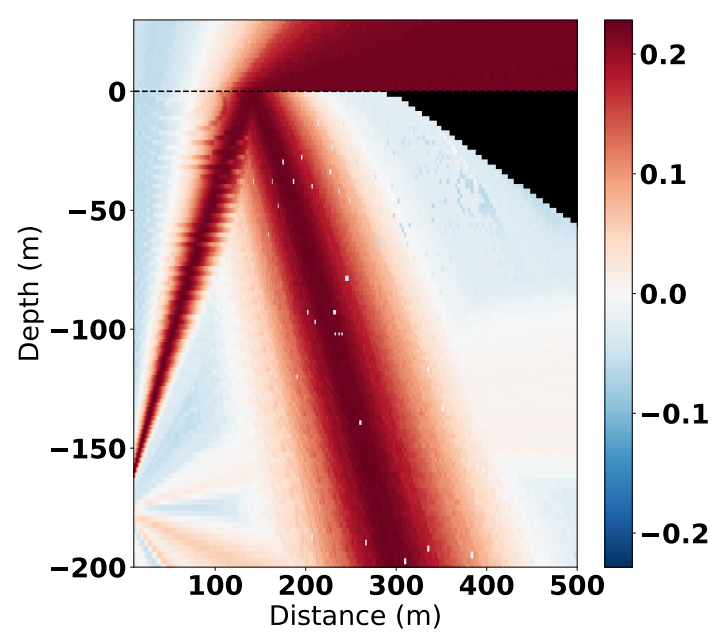

(b) A rooftop pulser event.

Figure 2: Two example distance vs. depth correlation maps, for both a local calibration pulser and a rooftop calibration pulser. 

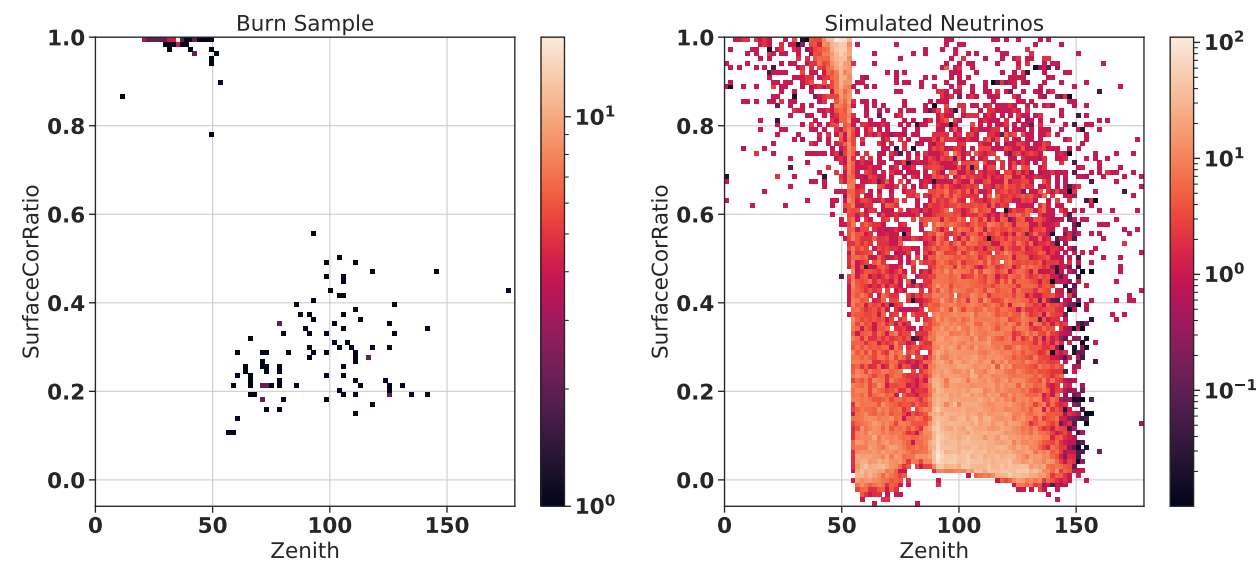

Figure 3: The two variables used to define the deep box: reconstructed zenith at the antenna, and the ratio of the maximum correlation within $10 \mathrm{~m}$ of the surface vs. the global maximum correlation. Only the highest correlating events are shown on the left plot, with a clear population of surface events in the upper right hand corner. On the right are all simulated events from a Kotera Mix Max flux.
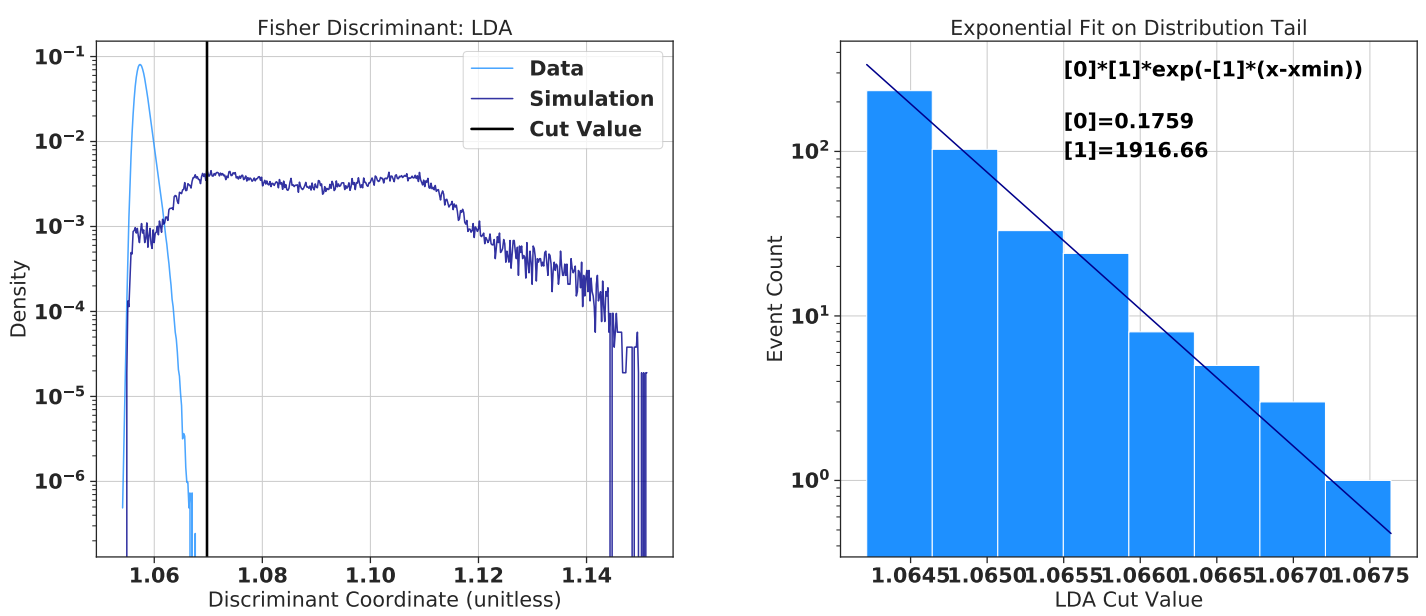

(a) Fisher Discriminant values for data vs. simulated neutri-(b) The tail of the background distribution, including the nos. exponential fit.

Figure 4: A comparison of the Fisher Discriminant from the 10\% sample against the simulated neutrino set. The cut value is determined by extrapolating the exponential fit and optimizing for the best Feldman-Cousins $90 \%$ upper limit [2]. 


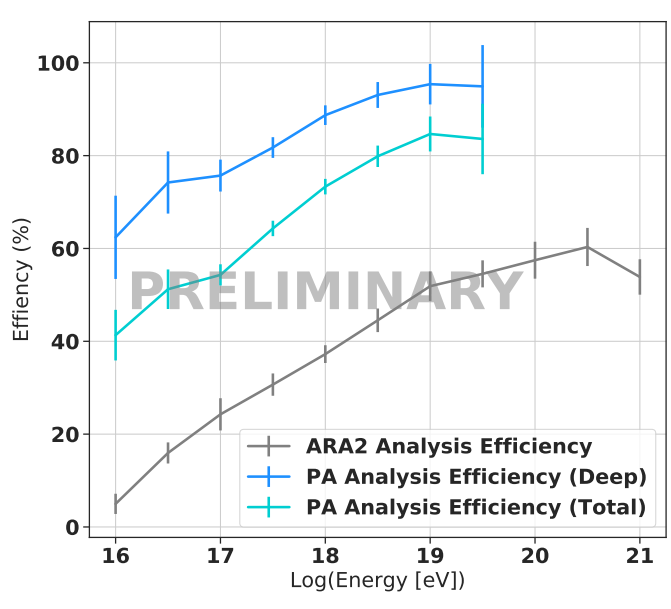

(a) Efficiency vs. Energy.

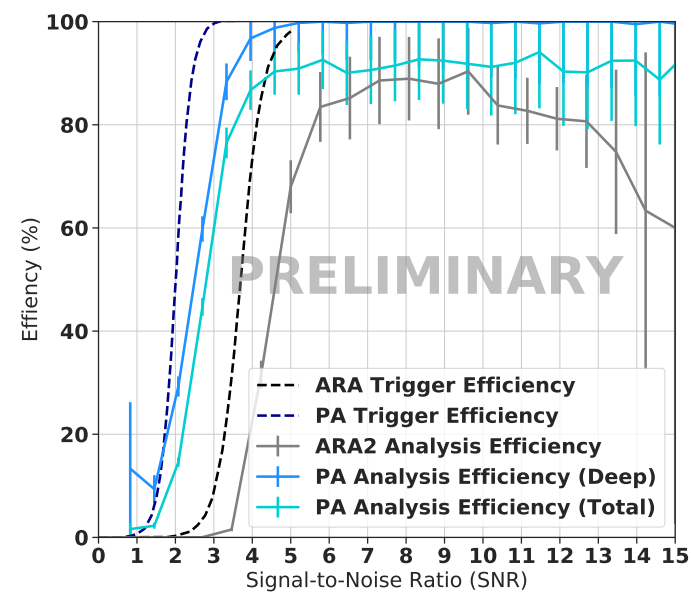

(b) Efficiency vs. SNR.

Figure 5: Analysis efficiency of this analysis compared to the most recent ARA Station 2 efficiency, scaled to have equal definitions. The blue curve is the efficiency on neutrinos in the deep region; the red curve assumes no shallow region neutrinos are recoverable, a conservative estimate that causes a $21 \%$ loss in efficiency over all energies. This analysis is efficient on events with low SNR that the traditional ARA trigger is not sensitive to.

allowed. The boundary for the deep region was set to 57 degrees, a conservative boundary from considering Snell's law at a depth of $20 \mathrm{~m}$ below the surface. This was chosen to avoid impacting cosmic ray events, which are expected to penetrate to $20 \mathrm{~m}$ maximally [6].

The second variable is the ratio of the correlation within $10 \mathrm{~m}$ of the surface and the global maximum correlation. This also strongly correlates with surface events in the $10 \%$ sample. True surface events may correlate to both the surface and the deep region (as shown in the rooftop pulser correlation map in Figure 2b) but the maximum within $10 \mathrm{~m}$ of the surface should be equal or comparable to the global maximum. The boundary on this ratio for the deep region was set to 0.5 , corresponding to signals in which the global maximum was more than twice that of the correlation within $10 \mathrm{~m}$ of the surface. All candidate surface events in the $10 \%$, as well as a population of surface calibration events, fall comfortably into the shallow region using this method.

\subsection{Designing Cuts}

There are two types of events that are universally removed from the analysis: calibration pulser events and software trigger events. ARA Station 5 is equipped with a local calibration pulser on its own string about $20 \mathrm{~m}$ away, which pulses on the GPS second, and thus can be easily removed with a trigger time cut, which is $99.984 \%$ efficient. Occasionally, the calibration pulser is operated in a mode that is not tied to the GPS time; these runs were tagged as calibration runs and removed from the livetime. In total, there were 13 of these calibration runs for a total of 1.6 days of livetime and all were removed. Software trigger events, which occur once per second and are not RF triggered, were also removed from the analysis. For each run, the software triggers were only used to calculate the expected RMS of each channel. 


\begin{tabular}{||cccc||}
\hline Cut Name & Events Remaining & Background Estimate & Simulation Efficiency \\
\hline \hline None & $6,005,122$ & 0 & $100 \%$ \\
\hline Cal Pulser Gate Flag & $4,423,436$ & 0 & $99.984 \%$ \\
\hline Cal Pulser Geometry Cut & $4,411,686$ & 0.009 & $99.644 \%$ \\
\hline Software Trigger Cut & $4,014,776$ & 0 & $100 \%$ \\
\hline Fisher Discriminant & 0 & $0.0865[0.0494,0.1500]$ & $86.58 \%$ \\
\hline Total & 0 & $0.0955[0.0584,0.1590]$ & $86.25 \%$ \\
\hline
\end{tabular}

Table 1: Table of cuts and background estimates from the $10 \%$ burn sample.

Throughout the year, there were a few periods with especially high backgrounds, often corresponding to station activity. These runs were identified by comparing the number of outliers in a distribution of maximum correlation to the expected tail of events in the full sample. If there were two or more outlier events in the $10 \%$ sample of a given run, that run was removed from the livetime. A total of six runs were removed due to meeting this threshold.

After the quality cuts were applied, the population of events left in the $10 \%$ data sample in the deep region had the qualities expected of thermal noise. A Fisher discriminant was then used to characterize the thermal sample against simulated Kotera Mix Max neutrinos [5]. The list of variables used as input to the Fisher discriminant are: average SNR, maximum correlation, various impulsivity measures, peak power of the coherently summed waveform, SNR of the coherently summed waveform, location of peak power within the waveform, and the correlation with the dual ARA5 / Phased Array channel. Three separate correlation maps were generated for each event, corresponding to direct, refracted, and combination paths, and all three maps were used to generate analysis quantities. The distribution of the Fisher discriminant is shown in Figure 4.

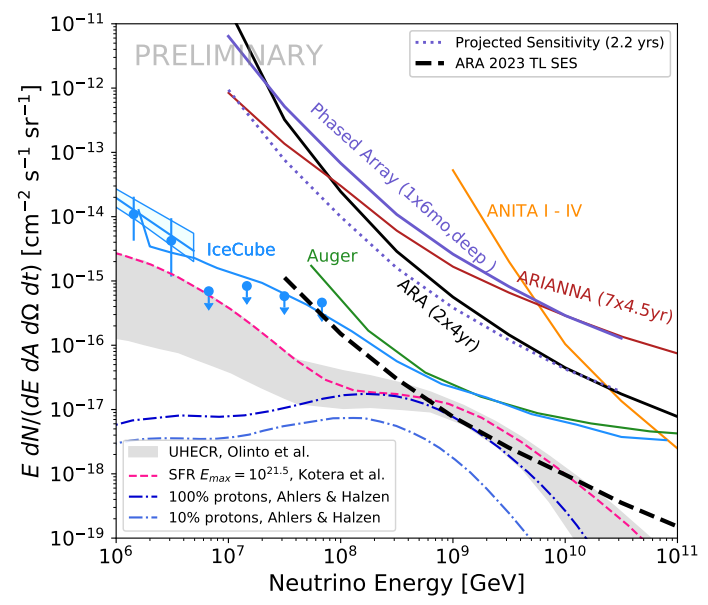

Figure 6: The limit from the phased array analysis, using six months of livetime, along with the projected sensitivity from the entire available livetime of 2.2 years.

\section{Results and Conclusions}

The final cuts and background estimate for the deep region are shown in Table 1. As a precaution, the region between 52-57 degrees in zenith was burned and unblinded to ensure no leakage from the surface into the deep box. In this region, we observe no events passing all cuts. After unblinding the deep region only, we observe one event on a background of $0.09_{-0.0316}^{+0.069}$. This event was triggered separately by both the traditional ARA trigger and the phased array trigger, and 
is consistent with background with a p-value of 0.107. The event does not appear to be a thermal noise fluctuation and is still under investigation. The event is included in the limit set in Figure 6.

While only the deep region of the dataset has been unblinded, the analysis efficiency is already higher than previous ARA analyses even in the scenario where the surface region is not usable. Additionally, the analysis is efficient on much lower SNR signals; in fact, events are kept through the analysis that a traditional ARA station would not trigger on. We are optimistic that the improvements shown here are transferable to future in-ice radio experiments as well.

\section{References}

[1] G.A. Askaryan. "Excess negative charge of an electron-photon shower and its coherent radio emission". In: Sov.Phys.JETP (1961).

[2] Gary J. Feldman and Robert D. Cousins. "Unified approach to the classical statistical analysis of small signals". In: Phys. Rev. D 57 (7 Apr. 1998), pp. 3873-3889.

[3] D. Saltzberg et. al. "Observation of the Askaryan Effect: Coherent Microwave Cherenkov Emission from Charge Asymmetry in High Energy Particle Cascades". In: Phys.Rev.Lett. 86 (2001).

[4] J. Klein and A. Roodman. "BLIND ANALYSIS IN NUCLEAR AND PARTICLE PHYSICS". In: Annual Review of Nuclear and Particle Science 55 (2005).

[5] K. Kotera and A. V. Olinto. "The Astrophysics of Ultrahigh-Energy Cosmic Rays”. In: Annual Review of Astronomy and Astrophysics 49 (Sept. 2011), pp. 119-153.

[6] K. D. de Vries et al. "Coherent Cherenkov Radiation from Cosmic-Ray-Induced Air Showers”. In: Phys. Rev. Lett. 107 (6 Aug. 2011), p. 061101.

[7] The ARA Collaboration. "First Constraints on the Ultra-High Energy Neutrino Flux from a Prototype Station of the Askaryan Radio Array". In: Phys. Rev. D. 70 (2015).

[8] David Besson Ilya Kravchenko and Josh Meyers. "In situ index-of-refraction measurements of the South Polar firn with the RICE detector". In: Cambridge University Press (2017).

[9] P. W. Gorham et. al. "Constraints on the diffuse high-energy neutrino flux from the third flight of ANITA”. In: Phys. Rev. D. 98 (2018).

[10] P. Allison et. al. "Design and Performance of an Interferometric Trigger Array for Radio Detection of High-Energy Neutrinos”. In: Nucl. Instrum. Methods Phys. Res 930 (2019).

[11] P.W. Gorham et. al. "Constraints on the ultra-high energy cosmic neutrino flux from the fourth flight of ANITA”. In: Phys. Rev. D. 99 (2019).

[12] P. Allison et. al. "Constraints on the Diffuse Flux of Ultra-High Energy Neutrinos from Four Years of Askaryan Radio Array Data in Two Stations". In: Phys. Rev. D. 102 (2020).

[13] The IceCube-Gen2 Collaboration. IceCube-Gen2: The Window to the Extreme Universe. Tech. rep. Aug. 2020, p. 93.

[14] A. Anker et. al. "Targeting ultra-high energy neutrinos with the ARIANNA experiment". In: Advances in Space Research 64 (2019) 2595-2609 (). 


\section{Full Authors List: ARA Collaboration}

P. Allison ${ }^{1}$, S. Archambault ${ }^{2}$, J.J. Beatty ${ }^{1}$, M. Beheler-Amass ${ }^{3}$, D.Z. Besson ${ }^{4,5}$, M. Beydler ${ }^{3}$, C.H. Chen ${ }^{6}$, Y.C. Chen ${ }^{6}$, P. Chen ${ }^{6}$, B.A. Clark ${ }^{7}$, W. Clay $^{8}$, A. Connolly ${ }^{1}$, L. Cremonesi ${ }^{9}$, P. Dasgupta ${ }^{10}$, J. Davies ${ }^{9}$, S. de Kockere ${ }^{11}$, K.D. de Vries ${ }^{11}$, C. Deaconu ${ }^{8}$, M. A. DuVernois ${ }^{3}$, J. Flaherty ${ }^{1}$, E. Friedman ${ }^{12}$, R. Gaior ${ }^{2}$, J. Hanson ${ }^{13}$, K. Hanson ${ }^{3}$, N. Harty ${ }^{14}$, B. Hendricks ${ }^{15,16}$, K.D. Hoffman ${ }^{12}$,

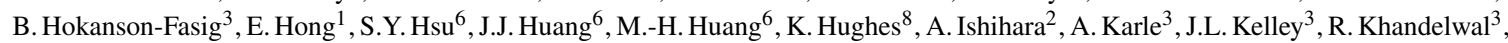
K.-C. Kim ${ }^{12}$, M.-C. $\mathrm{Kim}^{2}$, I. Kravchenko ${ }^{17}$, R. Krebs ${ }^{15,16}$, Y. Ku ${ }^{15,16}$, C.Y. Kuo ${ }^{6}$, K. Kurusu ${ }^{2}$, H. Landsman ${ }^{18}$, U.A. Latif ${ }^{4,11}$,

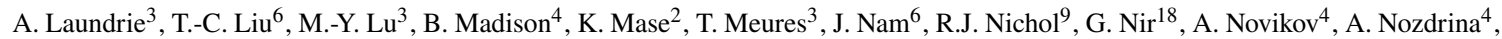
E. Oberla ${ }^{8}$, A. ÓMurchadha ${ }^{3}$, J. Osborn ${ }^{17}$, Y. Pan $^{14}$, C. Pfendner ${ }^{19}$, N. Punsuebsay ${ }^{14}$, J. Roth ${ }^{14}$, P. Sandstrom ${ }^{3}$, D. Seckel ${ }^{14}$, Y.S. Shiao ${ }^{6}$, A. Shultz ${ }^{4}$, D. Smith ${ }^{8}$, S. Toscano ${ }^{10}$, J. Torres $^{1}$, J. Touart ${ }^{12}$, N. van Eijndhoven ${ }^{11}$, G.S. Varner ${ }^{20}$, A. Vieregg ${ }^{8}$, M.-Z. Wang ${ }^{6}$, S.-H. Wang 6 , Y.H. Wang ${ }^{6}$, S.A. Wissel ${ }^{15,16,21,22}$, S. Yoshida ${ }^{2}$, R. Young ${ }^{4}$

${ }^{1}$ Dept. of Physics, Center for Cosmology and AstroParticle Physics, The Ohio State University, Columbus, OH 43210

${ }^{2}$ Dept. of Physics, Chiba University, Chiba, Japan

${ }^{3}$ Dept. of Physics, University of Wisconsin-Madison, Madison, WI 53706

${ }^{4}$ Dept. of Physics and Astronomy, University of Kansas, Lawrence, KS 66045

${ }^{5}$ Moscow Engineering Physics Institute, Moscow, Russia

${ }^{6}$ Dept. of Physics, Grad. Inst. of Astrophys., Leung Center for Cosmology and Particle Astrophysics, National Taiwan University, Taipei, Taiwan

${ }^{7}$ Dept. of Physics and Astronomy, Michigan State University, East Lansing, Michigan 48824

${ }^{8}$ Dept. of Physics, Enrico Fermi Institue, Kavli Institute for Cosmological Physics, University of Chicago, Chicago, IL 60637

${ }^{9}$ Dept. of Physics and Astronomy, University College London, London, United Kingdom

${ }^{10}$ Université Libre de Bruxelles, Science Faculty CP230, B-1050 Brussels, Belgium

${ }^{11}$ Vrije Universiteit Brussel, Brussels, Belgium

${ }^{12}$ Dept. of Physics, University of Maryland, College Park, MD 20742

${ }^{13}$ Dept. Physics and Astronomy, Whittier College, Whittier, CA 90602

${ }^{14}$ Dept. of Physics, University of Delaware, Newark, DE 19716

${ }^{15}$ Center for Multi-Messenger Astrophysics, Institute for Gravitation and the Cosmos, Pennsylvania State University, University Park, PA 16802

${ }^{16}$ Dept. of Physics, Pennsylvania State University, University Park, PA 16802

${ }^{17}$ Dept. of Physics and Astronomy, University of Nebraska, Lincoln, Nebraska 68588

${ }^{18}$ Weizmann Institute of Science, Rehovot, Israel

${ }^{19}$ Dept. of Physics and Astronomy, Denison University, Granville, Ohio 43023

${ }^{20}$ Dept. of Physics and Astronomy, University of Hawaii, Manoa, HI 96822

${ }^{21}$ Dept. of Astronomy and Astrophysics, Pennsylvania State University, University Park, PA 16802

${ }^{22}$ Physics Dept., California Polytechnic State University, San Luis Obispo, CA 93407

\section{Acknowledgements}

We thank the National Science Foundation Office of Polar Programs and Physics Division for their generous support through NSF OPP-902483, Grant NSF OPP-1002483, Grant NSF 1607555, Grant NSF OPP-1359535, Grant NSF OPP-1404212, and Grant NSF OPP-2013134, and Grant NSF 2019597. K. Hughes thanks the NSF for support through DGE-1746045. 